\title{
A contemporary review of peripartum cardiomyopathy
}

\author{
Authors: Peysh A Patel, ${ }^{A, *}$ Ashwin Roy, ${ }^{B, *}$ Rabeia Javid ${ }^{C, *}$ and John AW Dalton ${ }^{D}$
}

Peripartum cardiomyopathy reflects the presence of cardiac failure in the absence of determinable heart disease and occurs in late third trimester of pregnancy or up to 6 months postpartum. A full understanding of pathophysiological mechanisms is lacking, but excess prolactin levels, haemodynamic alterations, inflammation and nutritional deficiencies have all been implicated. Its clinical presentation has distinct overlap with physiological alterations in healthy pregnancy and this presents a diagnostic challenge. However, echocardiography can provide significant benefit in accurate assessment and narrowing of differentials. Pharmacotherapy is broadly aligned with established guidelines for cardiac failure, but specific therapies are indicated for treatment of clinical sequelae. Moreover, an individualistic approach is required based on clinical context to manage delivery. Further research appears imperative to optimise management strategies and reduce disease burden.

KEYWORDS: Cardiac failure, cardiomyopathy, peripartum, pregnancy, ventricular dysfunction

\section{Introduction}

Heart disease represents the leading overall cause of maternal death and is steadily rising. In the last two triennia, the all-cause cardiovascular mortality rate was 2 per 100,000 maternities according to the MBRRACE-UK (Mothers and Babies: Reducing Risk through Audits and Confidential Enquiries across the UK) collaboration. ${ }^{1}$ Thrombosis and thromboembolism constitute the predominant burden of disease. In comparison, the true morbidity and mortality associated with peripartum cardiomyopathy (PPCM) remains unknown because of poor index of suspicion and late recognition. This review provides a contemporary overview of pathophysiology, clinical presentation, diagnosis and management of PPCM.

Authors: A cardiology registrar and clinical research fellow, Leeds General Infirmary, Leeds, UK; ${ }^{B}$ core medical trainee, The Christie, Manchester, UK; ' Cacute medicine registrar, Dewsbury District Hospital, Dewsbury, UK; ${ }^{D}$ obstetrics and gynaecology registrar and clinical research fellow, Leeds General Infirmary, Leeds, UK; * joint first authors

\section{Epidemiology}

Accurate assessment of the epidemiological profile of PPCM has been challenging because of limitations arising from ambiguities in diagnosis and variability in reporting standards. The incidence of disease in the UK is rare and estimated as 1 in 5,000 to 1 in 10,000 live births. ${ }^{2}$ In the USA, an overall incidence of 1 in 968 live births has been reported from the National Inpatient Database, collated between 2004 and 2011. ${ }^{3}$ Data derived from Asia and South Africa suggest an incidence of 1 in 1,000 live births, ${ }^{4}$ with an even lower incidence of 1 in 6,000 live births quoted in Japan. ${ }^{5}$ There appears to be a racial predisposition, with Afro-Caribbean women appearing to have the highest incidence (a 16-fold increased risk). ${ }^{6}$ This may be attributable to environmental, ecological, cultural, genetic, puerperal and post-puerperal practices.

\section{Pathophysiology}

A full comprehension of pathophysiological mechanisms that underpin PPCM appears to be lacking. The observed geographical variation suggests potential genetic predisposition, with known familial clustering providing further support to this notion. ${ }^{7}$ There appears to be an association with the parathyroid hormone-like hormone $(P T H L H)$ gene, which modulates vascular homeostasis via calcium channel agonisation. ${ }^{8}$ Moreover, the TTN gene, which encodes the sarcomeric protein titin, has also been implicated in pathogenesis. ${ }^{9}$

A novel hypothesis relates to excess prolactin production, which is associated with enhanced intravascular volumes, suppressed response to angiotensin and increased circulating erythropoietin levels. ${ }^{10}$ An exploration of unifying mechanisms is provided later in this article and is summarised in Fig 1. Alternate suggestions indicate that PPCM occurs secondary to haemodynamic perturbations in the context of pregnancy. It is evident that intravascular volume and red cell mass is enhanced with a concurrent increase in preload and cardiac output via the Frank-Starling mechanism. It has been postulated that these physiological adaptations may also be implicated in the cardiomyopathic phenotype. ${ }^{11}$ However, these changes typically occur during the first and second trimesters and not in the peripartum period. Even when additional stresses implicated in delivery, (such as caesarean section), have been accounted for, propensity for developing PPCM does not appear to be significantly raised. 


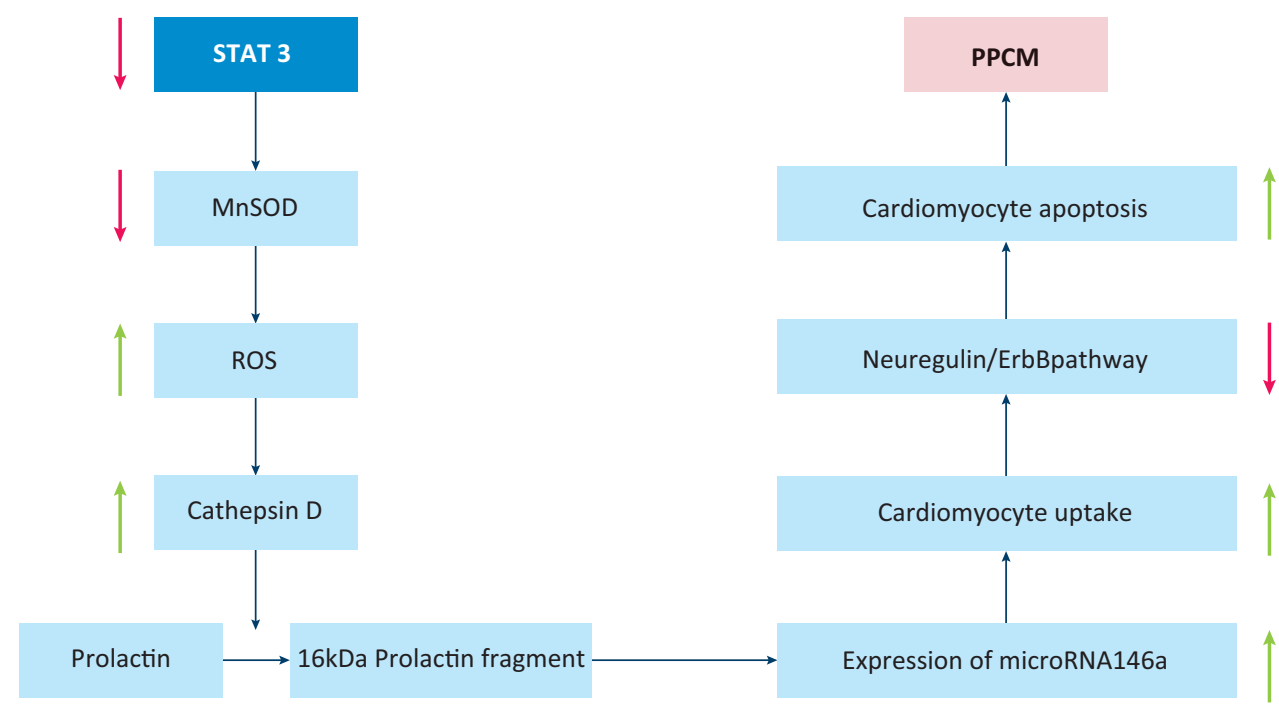

Fig 1. Schema representing excess prolactin theory in peripartum cardiomyopathy (PPCM). MnSOD = manganese superoxide dismutase; ROS = reactive oxygen species; STAT3 = signal transducer and activator of transcription 3

Conflicting reports implicate myocarditis as a potential trigger for PPCM. ${ }^{10}$ The underlying mechanism may relate to systemic viral illness that results in secondary inflammation of the myocardial interstitium. Studies using endomyocardial biopsies have shown infiltrates containing parvovirus B19 and coxsackie virus ${ }^{12}$ although, overall, results have been inconclusive. Other proposed aetiologies include michochimerism and nutritional deficiencies in iron and selenium. Fetal microchimerism describes the presence of fetal cells as a consequence of previous pregnancy. In these cohorts, a higher incidence of PPCM is observed and may relate to the triggering of an autoimmune response. ${ }^{13}$ Iron deficit has been implicated, potentially, in the context of high-output cardiac failure. ${ }^{10}$ Lastly, selenium deficiency was considered as a potential cause after an endemic of cardiomyopathy (Keshan disease) in China. ${ }^{14}$ Selenium mediates specific enzymes, namely selenoproteins, that normally act to reduce oxidative stress in cardiomyocytes.

\section{Clinical presentation}

Onset is usually in the first month following delivery, but may occur in the late third trimester and up to 6 months postnatally. PPCM is associated with various risk factors, which are listed in Box 1. The diagnostic challenge is perpetuated by nonspecific features on clinical presentation, which are difficult to differentiate from adaptive responses implicated in normal pregnancy. Broadly, symptoms of PPCM correlate reasonably with that of congestive cardiac failure. Hence, patients may report dyspnoea, orthopnoea and peripheral oedema. Nonspecific symptoms of fatigue and generalised malaise often coexist. More rarely, patients may describe symptoms of neurological deficit secondary to systemic embolisation in the context of cardiogenic thrombus.

Examination features may include sinus tachycardia, elevated jugular venous pressure and peripheral congestion. Features of left ventricular dilation may include a third heart sound (S3) from rapid ventricular filling, displaced apex beat and a pansystolic murmur consistent with functional mitral regurgitation. However, S3 is a normal finding in pregnancy and systolic flow murmurs are audible in the majority of patients because of the hyperdynamic circulation. In rarer instances, PPCM may present more acutely with respiratory failure attributable to pulmonary congestion or cardiogenic shock without prior symptoms to alert clinicians.

\section{Differential diagnosis}

PPCM is defined as idiopathic cardiac failure occurring in the absence of determinable heart disease, and within the time period outlined earlier. ${ }^{15}$ Hence, correct diagnosis relies on appropriate exclusion of other aetiologies of cardiac failure. A number of pre-existing conditions may be unmasked in pregnancy and can present similarly to that of PPCM. Both idiopathic and familial dilated cardiomyopathy - which typically manifest during periods of increased cardiac workload, namely in the first and third trimesters - should

Box 1. Risk factors in peripartum cardiomyopathy
$>$ Increased maternal age
$>$ Afro-Caribbean race
$>$ Multiparity
> Multiple pregnancies
$>$ Hypertensive disease:
- essential hypertension
- pregnancy-induced hypertension
- pre-eclampsia
$>$ Lower socio-economic status
$>$ Metabolic dysregulation:
- obesity
- pre-diabetes
- diabetes 
be considered. For this reason, it is particularly difficult to distinguish true PPCM from third trimester decompensation in the context of previously undiagnosed dilated cardiomyopathy. Pre-existing valvular and congenital heart disease may also be implicated, but again, is a diagnostic challenge. A thorough antenatal booking visit is often helpful in identifying preexistent cardiac disease and precipitating referral for further investigations in a combined cardiac antenatal setting.

Hypertensive heart disease in the context of pre-eclampsia may present as cardiac failure and, as a consequence, stringent blood pressure assessment throughout pregnancy is warranted. High-output cardiac states, such as anaemia and thyrotoxicosis, can be confidently excluded through blood profiling. More rarely, in patients with clear predisposition, there may be a manifestation of HIV-induced cardiomyopathy or ischaemic cardiomyopathy secondary to myocardial infarction. Lastly, pregnancy induces a pro-coagulant state and, therefore, a high index of suspicion for pulmonary embolism is required. A comprehensive review of the patient's history and clinical presentation is of paramount importance.

\section{Investigations}

Initial blood profiling is helpful to exclude significant anaemia and active infection. Liver function tests may be deranged because of hepatic congestion secondary to cardiac failure although HELLP (haemolysis, elevated liver enzymes, low platelet count) syndrome in the context of pre-eclampsia can present similarly. B-type natriuretic peptide (BNP) levels are often raised in the context of both PPCM and normal pregnancy because of alterations in ventricular dimensions. ${ }^{16}$ Hence, isolated readings are not particularly helpful although serial measurements to monitor trends can provide an indication of response to therapy.

A 12-lead electrocardiogram is most often unremarkable, or shows sinus tachycardia and/or non-specific T-wave changes, which can equally be present in healthy pregnancy. Chest radiography may show cardiomegaly and/or features of congestion, including upper lobe diversion, pleural effusions and interstitial oedema.

Echocardiography is the investigation of choice in PPCM as it is well tolerated and without adverse effects. Diagnostic criteria include left ventricular ejection fraction (LVEF) $<45 \%$ (and/or fractional shortening $<30 \%$ ) and left ventricular end diastolic dimension (LVEDD) $>2.7 \mathrm{~cm} / \mathrm{m}^{2}{ }^{17}$ These are nonspecific features, as they can exist in patients with ventricular dysfunction of any aetiology. PPCM is, therefore, a diagnosis of exclusion, but, nonetheless, echocardiography becomes of broad benefit in excluding differentials - including significant valvular disease.

Although echocardiography is widely available to clinicians, image quality is often compromised in pregnancy. Hence, cardiac magnetic resonance imaging is favoured in some centres to provide detailed assessment of anatomy and function. Conventionally, use is in the postpartum period. Lastly, endomyocardial biopsy has been performed in some centres, often as part of the workup for cardiac transplantation. ${ }^{18}$ However, its role is limited by poor diagnostic sensitivity. There may be histological evidence of inflammatory cells, cardiomyocyte hypertrophy and/or fibrotic deposition, but no pathognomonic features exist.

\section{Management}

\section{Timing of delivery}

The majority of cases occur postnatally. For those that present antenatally, timing of delivery requires a balanced approach to evaluate relative risks of deteriorating maternal condition versus fetal compromise secondary to prematurity. Hence, it is imperative to involve a multidisciplinary team of senior cardiologists, obstetricians, anaesthetists and neonatologists early in the decision-making process. Management should occur in a specialist high-risk obstetrics centre with experience in cardiac disease. In cases of deteriorating maternal and fetal wellbeing, maternal health ought to be prioritised. In the absence of overt symptoms consistent with cardiac failure, medical therapy and a spontaneous vaginal delivery is a reasonable strategy. Induction of labour is appropriate in refractory cases, or if there is an obstetric indication for fetal delivery. If delivery is required, a vaginal route is generally preferred because of the lower risk of adverse sequelae, including pulmonary embolism. ${ }^{19}$ Caesarean section is usually reserved for an obstetric indication or if there is severe maternal compromise.

Invasive monitoring of arterial and central venous pressure is recommended, with consideration of temporary suspension of heparin prophylaxis to permit use of regional anaesthesia. This has adjunct benefits in reducing maternal pain and the tachycardia response. Positioning the mother in a $30^{\circ}$ left tilt is paramount to minimising risk of aorto-caval compression. General anaesthesia may be considered if the patient is unable to lie supine. In order to negate the cardiovascular effects of the Valsalva manoeuvre associated with prolonged pushing in the second stage of labour, instrumental delivery can be used to limit the active phase. Uterotonic drugs should be used with caution in patients with PPCM; ergometrine and oxytocin boluses should be avoided because of their cardiovascular effects, but slow oxytocin infusion may be used for management of third stage of labour. ${ }^{20}$ Carboprost can cause severe bronchospasm, hypertension, pulmonary congestion and cardiogenic shock, making it equally unsuitable for use in most instances. ${ }^{21}$ In cases of iatrogenic preterm birth due to maternal deterioration, intramuscular corticosteroids are generally administered between 24 and 36 weeks gestational age to promote fetal lung maturation. ${ }^{22}$ A clear multidisciplinary delivery plan should be available in the maternity notes for staff to follow once the mother presents in labour. Inadequate communication between specialties has been highlighted by MBRRACE-UK as a significant contributor to cardiac-related maternal deaths. ${ }^{23}$

\section{Medical therapy}

Management of PPCM relies on the general guidelines provided by the European Society of Cardiology (ESC) for treatment of systolic cardiac failure. ${ }^{17}$ A tailored approach is required throughout with selection of agents that are tolerable and have better safety profiles in the context of pregnancy and lactation. Broadly speaking, the aim of therapy is to stabilise the patient by controlling volume status and regressing pathological neurohumoral responses. Duration of pharmacotherapy depends on the extent of recovery of ventricular function. A broad overview of options pertaining to medical therapy in PPCM is provided in Table 1. 
Table 1. Overview of potential medical therapies in peripartum cardiomyopathy

\begin{tabular}{|c|c|c|c|c|}
\hline Drug & Safety profile in pregnancy & $\begin{array}{l}\text { Safety profile } \\
\text { in lactation }\end{array}$ & $\begin{array}{l}\text { Absence of full recovery of LV } \\
\text { function }\end{array}$ & $\begin{array}{l}\text { Fully recovered LV } \\
\text { function }\end{array}$ \\
\hline Diuretics & Safe & $\begin{array}{l}\text { Thiazides best } \\
\text { studied }\end{array}$ & Continue only for symptomatic relief & $\begin{array}{l}\text { Discontinue when } \\
\text { asymptomatic }\end{array}$ \\
\hline ACE inhibitors & $\begin{array}{l}\text { Risk of damage to fetal renal } \\
\text { system }\end{array}$ & Relatively safe & Essential & $\begin{array}{l}\text { Continue for at least } \\
12 \text { months }\end{array}$ \\
\hline ARB & Teratogenic & $\begin{array}{l}\text { Limited data so } \\
\text { best avoided }\end{array}$ & $\begin{array}{l}\text { Recommended in patients who cannot } \\
\text { tolerate ACE inhibitors }\end{array}$ & $\begin{array}{l}\text { Continue for at least } \\
12 \text { months }\end{array}$ \\
\hline Vasodilators & $\begin{array}{l}\text { Use with caution, may precipitate } \\
\text { uterine hypoperfusion }\end{array}$ & Safe & Continue only for symptomatic relief & $\begin{array}{l}\text { Discontinue when } \\
\text { asymptomatic }\end{array}$ \\
\hline Beta blockers & $\begin{array}{l}\text { Safe, with metoprolol } \\
\text { recommended }\end{array}$ & Safe & Essential & $\begin{array}{l}\text { Continue for at least } \\
12 \text { months }\end{array}$ \\
\hline Ivabradine & No data so best avoided & $\begin{array}{l}\text { No data so } \\
\text { best avoided }\end{array}$ & $\begin{array}{l}\text { Alternative to beta blocker therapy (if } \\
\text { NSR), adjunct therapy if HR suboptimal }\end{array}$ & $\begin{array}{l}\text { Discontinue when } \\
\text { HR<75 bpm }\end{array}$ \\
\hline MRA & No data so best avoided & $\begin{array}{l}\text { Limited data so } \\
\text { best avoided }\end{array}$ & $\begin{array}{l}\text { Recommended for patients with } \\
\text { LVEF }<40 \%\end{array}$ & $\begin{array}{l}\text { Continue for at least } \\
6 \text { months }\end{array}$ \\
\hline
\end{tabular}

$\mathrm{ACE}$ = angiotensin-converting enzyme; $\mathrm{ARB}$ = angiotensin II receptor blocker; $\mathrm{HR}=$ heart rate; $\mathrm{LVEF}=$ left ventricular ejection fraction; $\mathrm{MRA}=$ mineralocorticoid receptor antagonists; NSR = normal sinus rhythm

Diuretics can be safely administered in pregnancy and relieve symptoms by reducing intravascular volume and right ventricular filling (preload). Close monitoring for electrolyte imbalance is required. Angiotensin-converting enzyme inhibitors and angiotensin II receptor blockers operate primarily through reduction of afterload. However, they are contraindicated in all trimesters of pregnancy because of their teratogenic potential; ${ }^{24}$ vasodilatory agents, such as nitrates and hydralazine, can be utilised as effective alternatives. ${ }^{25}$ Beta blockers are a component of conventional cardiac failure therapy and can be safely administered during pregnancy and in the postpartum period. Metoprolol is most commonly prescribed because of its extensive use in clinical settings and shorter half-life, which enables safe assessment of tolerability. In patients with intolerance or suboptimal rate control despite use of beta blocker therapy, ivabradine can be considered as per findings from registries in the ESC Euro observational programme. ${ }^{26}$ This has negative chronotropic effects via suppression of the I $f$ channel in the sinoatrial node. It does not compromise blood pressure, due to compensatory increase in stroke volume, but is ineffective in the context of coexistent atrial fibrillation. Mineralocorticoid receptor antagonists, such as spironolactone and eplerenone, are generally recommended in patients with $\mathrm{LVEF}<40 \% .{ }^{26}$ Inotropic support is reserved for patients with haemodynamic instability in the context of acute decompensation.

\section{Treatment of sequelae}

Ventricular dilatation and dysfunction in the context of PPCM results in blood stasis and this can predispose to thrombus formation in the ventricles. Thromboembolism is recognised as the most serious complication in patients with PPCM, arising in around $6 \%$ of cases. ${ }^{4}$ Low molecular weight heparin is the preferred therapeutic agent in the antepartum period because of its short half-life, rapid reversibility and adequate safety profile in pregnancy. Postpartum, warfarin or novel oral anticoagulant therapy can be used and should be maintained until pregnancyrelated hypercoagulability resolves.

Ventricular remodelling can also provide substrate for dysrhythmias. Indeed, up to $25 \%$ of mortality cases in PPCM are secondary to lethal tachyarrhythmias. ${ }^{27}$ However, as a significant proportion demonstrate good functional recovery, wearable cardioverter-defibrillators (LifeVest) can be potentially considered as a bridge to definitive decision making with regards to primary prevention implantable cardioverter defibrillator. ${ }^{28}$ All patients who survive a cardiac arrest secondary to a tachyarrhythmia should be considered for a secondary prevention implantable cardioverter defibrillator as per established guidelines. $^{29}$

In patients with acute cardiac failure and features of decompensation that are refractory to medical intervention, mechanical circulatory support can be considered. This includes use of intra-aortic balloon pump counter-pulsation, ventricular assist devices and extracorporeal systems. ${ }^{24}$ However, these patients are inherently high risk and, at such an advanced stage, prognosis is dismal. Only around 6\% of patients on mechanical support make a full recovery and nearly half will require a cardiac transplant to be considered. Data suggest that women with PPCM requiring circulatory support have better survival outcomes in comparison with patients without PPCM, potentially attributable to their younger age and lower burden of comorbidities. However, those with PPCM who undergo a cardiac transplant appear to show reduced graft and age-adjusted survival, which may reflect higher propensity for allosensitisation. ${ }^{24}$

\section{Subsequent pregnancies}

Pre-pregnancy counselling is paramount as it is essential to outline the risks of recurrent cardiac failure and associated mortality. After discontinuation of pharmacotherapy, a 
surveillance echocardiogram is required at 6 months to ensure that ventricular function remains preserved. If there is persistent dysfunction, subsequent pregnancy is strongly discouraged because of a $25 \%$ risk of maternal death. ${ }^{30}$ Contraceptive advice should be provided in the puerperium prior to discharge and revisited at postnatal review.

\section{Current research}

Levosimendan is a positive inotrope and vasodilator that has been approved by the ESC for treatment of acute cardiac failure with cardiogenic shock if beta blockade is thought to be partly responsible. ${ }^{31}$ However, this agent has not shown any benefit in randomised controlled trials in terms of resolution of symptoms and improvements in ventricular function. ${ }^{32} \mathrm{~A}$ more recent, non-randomised prospective study has suggested a potential benefit for anti-tumour necrosis factor $\alpha$ therapy with pentoxyfylline ${ }^{33}$ although trends have not yet been corroborated in more established studies.

Preliminary studies have suggested an emerging indication for bromocriptine, which reduces prolactin levels, as a therapeutic agent in PPCM. ${ }^{34}$ It is based on the hypothesis that PPCM is associated with suppressed expression of signal transducer and activator of transcription 3 (STAT3). ${ }^{35}$ This is a transcription factor present in cardiomyocytes that mediates expression of genes, such as manganese superoxide dismutase, to protect against reactive oxygen species. A schematic overview of the underlying postulated link between prolactin and PPCM is provided in Fig 1. Interestingly, a pilot study has shown correlation between bromocriptine therapy and functional recovery of ventricular function at 6 months. ${ }^{36}$ However, a larger prospective cohort study showed that recovery was most significant in those concurrently administered angiotensinconverting enzyme inhibitor and beta blocker therapy, suggesting potential confounding effects. ${ }^{37}$ A multicentre randomised trial is currently ongoing to evaluate the safety profile and benefits of adjunct therapy with bromocriptine on morbidity and mortality in the context of PPCM. ${ }^{38}$

\section{Prognosis}

Overall, the prognosis of patients with PPCM remains reasonable, with around 50\% demonstrating echocardiographic resolution of ventricular impairment at 12 months. ${ }^{39}$ Despite this, overall mortality rates remain significant at up to $10 \%{ }^{40}$ Evidence of raised LVEDD, reduced fractional shortening, presence of cardiogenic thrombus and raised pulmonary artery pressures are associated with poorer long-term outcomes. ${ }^{41}$ ESC guidelines advocate the need to develop a novel biomarker that is specific to PPCM. Presently, pro-BNP is the only option commercially available to clinicians but it has poor specificity. Troponin levels appear to correlate inversely with LVEF, suggesting a possible role in guiding management. ${ }^{42}$

\section{Conclusions}

There have been concerted efforts in recent years to improve our understanding of mechanisms and pathophysiological processes implicated in PPCM. Nonetheless, many questions remain unresolved. PPCM continues to be a rare disease of pregnancy and its complex aetiology has not been fully elucidated. Moreover, no large-scale studies have been conducted to date. Limitations have been exacerbated by the significant heterogeneity in clinical presentation, diagnostic overlap with other precipitants of cardiac failure and the lack of a diagnostic marker specific to PPCM. Further research in this field is imperative to enhance understanding and enable directed treatment strategies to be incorporated into routine practice.

\section{Conflicts of interests}

The authors have no conflicts of interest to declare.

\section{Author contributions}

PAP: project design, drafting of manuscript. AR: drafting of manuscript, RJ: drafting of manuscript, JAWD: project design, critical revision.

\section{References}

1 MBRRACE-UK. Saving lives, improving mothers' care: surveillance of maternal deaths in the UK 2012-14 and lessons learned to inform maternity care from the UK and Ireland Confidential Enquiries in Maternal Deaths and Morbidity 2009-14. Oxford: MBRRACE-UK, 2016.

2 Adamson D, Dhanjal M, Nelson-Piercy C. Heart disease in pregnancy. Oxford: Oxford University Press, 2011:143-4.

3 Kolte D, Khera S, Aronow WS et al. Temporal trends in incidence and outcomes of peri-partum cardiomyopathy in the United States: a nationwide population based study. J Am Heart Association 2014;3:e001056.

4 Arany Z, Elkayam U. Peripartum cardiomyopathy. Circulation 2016;133:1397-409.

5 Lok SI, Kirkels JH, Klopping C et al. Peripartum cardiomyopathy: the need for a national database. Nether Heart J 2011;19:126-33.

6 Pearson JD, Veille JC, Rahimtoola S et al. National Heart Lung and Blood Institute and Office of Rare Diseases Workshop recommendations and Reviews. JAMA 2000;283:1183-8.

7 Ware JS, Li J, Mazaika E et al. Shared genetic predisposition in peripartum and dilated cardiomyopathies. N Engl J Med 2016;374:23341.

8 Horne BD, Rasmusson KD, Alharethi R et al. Genome-wide significance and replication of the chromosome 12p11.22 locus near the PTHLH gene for peripartum cardiomyopathy. Circ Cardiovasc Genet 2011;4:359-66.

9 Ware JS, Li J, Mazaika E et al. Shared genetic predisposition in peripartum and dilated cardiomyopathies. N Engl J Med 2016;374:23341.

10 Ntusi NB, Mayosi BM. Aetiology and risk factors of peripartum cardiomyopathy: a systematic review. Clin Res Cardiol 2015;104:911-7.

11 Ruys TP, Roos-Hesselink JW, Hall R et al. Heart failure in pregnant women with cardiac disease: data from the ROPAC. Heart 2014;100:231-8.

12 Bültmann BD, Klingel KN, Bauer M et al. High prevalence of viral genomes and inflammation in peripartum cardiomyopathy. Am J Obstet Gynecol 2005;193:363-5.

13 Ansari AA, Fett JD, Carraway RE et al. Autoimmune mechanisms as the basis for human peripartum cardiomyopathy. Clin Rev Allergy Immunol 2002;23:301-24.

14 Dasgupta S, Aly AM. Dilated Cardiomyopathy induced by chronic starvation and selenium deficiency. Case Rep Pediatr 2016;2016:8305895.

15 Demakis JG, Rahimtoola SH. Peripartum cardiomyopathy. Circulation 1971;44:964-8. 
16 Forster O, Hilfiker-Kleiner D, Ansari AA et al. Reversal of IFNgamma, oxLDL and prolactin serum levels correlate with clinical improvement in patients with peripartum cardiomyopathy. Eur J Heart Fail 2008;10:861-8.

17 Ponikowski P, Voors AA, Anker SD et al. 2016 ESC guidelines for the diagnosis and treatment of acute and chronic heart failure: the task force for the diagnosis and treatment of acute and chronic heart failure of the European Society of Cardiology (ESC). Eur Heart J 2016;37:2129-200.

18 Rizeq MN, Rickenbacher PR, Fowler MB et al. Incidence of myocarditis in peripartum cardiomyopathy. Am J Cardiol 1994;74:4747.

19 Gregory KD, Jackson S, Korst L et al. Cesarean versus vaginal delivery: whose risks? Whose benefits? Am J Perinatol 2012;29:7-18.

20 Tan J, Cao Q, He GL et al. Misoprostol versus ergometrine-oxytocin for preventing postpartum haemorrhage: a systematic review and meta-analysis of randomized controlled trials. J Evid Based Med 2016;9:194-204.

21 Chelmow D. Postpartum haemorrhage: prevention. BMJ Clin Evid 2011:1410.

22 Bolt RJ, van Weissenbruch MM, Lafeber HN et al. Glucocorticoids and lung development in the fetus and preterm infant. Pediatr Pulmonol 2001;32:76-91.

23 Freedman RL, Lucas DN. MBRRACE-UK: saving lives, improving mothers' care - implications for anaesthetists. Int J Obstet Anesth 2015;24:161-73.

24 Einav S, Varon J. Cardiomyopathy of pregnancy. Current Womens Health Rev 2016;12:3-13.

25 Davis M., Duvernoy C. Peripartum cardiomyopathy: current knowledge and future directions. Womens Health 2015;11:565-73.

26 Hilfiker-Kleiner D, Haghikia A, Nonhoff J et al. Peripartum cardiomyopathy: current management and future perspectives. Eur Heart J 2015;36:1090-7.

27 Honigberg M, Givertz MM. Arrhythmias in peripartum cardiomyopathy. Card Electrophysiol Clin 2015;7:309-17.

28 Duncker D, Haghikia A, Konig T et al. Risk for ventricular fibrillation in peripartum cardiomyopathy with severely reduced left ventricular function -value of the wearable cardioverter/defibrillator. Eur J Heart Fail 2014;16:1331-6.

29 Prior SG, Blonstrom-Lundqvist C, Mazzanti A et al. 2015 ESC guidelines for the management of patients with ventricular arrhythmias and the prevention of sudden cardiac death. Eur Heart J 2015;36:2757-9.

30 Elkayam U, Tummala PP, Rao K. Maternal and fetal outcome of subsequent pregnancies in women with peripartum cardiomyopathy. N Engl J Med 2001;344:1567-71.
31 Fruhwald S, Pollesello P, Fruhwald F. Advanced heart failure: an appraisal of the potential of levosimendan in the end-stage scenario and some related ethical considerations. Expert Rev Cardiovasc Ther 2016;14:1335-47.

32 Pierrakos C, Velissaris D, Franchi F et al. Levosimendan in critical illness: a literature review. J Clin Med Res 2014;6:75-85.

33 Sliwa K, Skudicky D, Candy G et al. The addition of pentoxifylline to conventional therapy improves outcome in patients with peripartum cardiomyopathy. Eur J Heart Fail 2002;4:305-9.

34 Hilfiker-Kleiner D, Kaminski K, Podewski E et al. A cathepsin $\mathrm{D}$-cleaved $16 \mathrm{kDa}$ form of prolactin mediates postpartum cardiomyopathy. Cell 2007;128:589-600.

35 Halkein J, Tabruyn SP, Ricke-Hoch M et al. MicroRNA-146a is a therapeutic target and biomarker for peripartum cardiomyopathy. J Clin Invest 2013;123:2143-54.

36 Sliwa K, Hilfiker-Kleiner D, Petrie MC et al. Current state of knowledge on aetiology, diagnosis, management, and therapy of peripartum cardiomyopathy: a position statement from the Heart Failure Association of the European Society of Cardiology Working Group on peripartum cardiomyopathy. Eur J Heart Fail 2010;12:767-78.

37 Haghikia A, Podewski E, Libhaber E. Phenotyping and outcome on contemporary management in a German cohort of patients with peripartum cardiomyopathy. Basic Res Cardiol 2013;108:366.

38 Haghikia A, Podewski E, Berliner D et al. Rationale and design of a randomized, controlled multicentre clinical trial to evaluate the effect of bromocriptine on left ventricular function in women with peripartum cardiomyopathy. Clin Res Cardiol 2015;104:911-7.

39 Bhattacharyya A, Basra SS, Sen P et al. Peripartum cardiomyopathy: a review. Tex Heart Inst J 2012;39:8-16.

40 Felker GM, Jaeger CJ, Klodas E et al. Myocarditis and long-term survival in peripartum cardiomyopathy. Am Heart J 2000;140:78591.

41 Fett JD, Fristoe KL, Welsh SN. Risk of heart failure relapse in subsequent pregnancy among peripartum cardiomyopathy mothers. Int $J$ Gynaecol Obstet 2010;109:34-6.

42 Hu CL, Li YB, You YG et al. Troponin T measurement can predict persistent left ventricular dysfunction in peripartum cardiomyopathy. Heart 2007;93:488-90.

Address for correspondence: Dr Peysh Patel, Department of Cardiology, Leeds General Infirmary, Great George Street, Leeds LS1 3EX, UK.

Email:p.a.patel@leeds.ac.uk 